\title{
Electromagnetic Navigational Bronchoscopy
}

\author{
Jeffrey Port, $\mathrm{MD}^{1}$ Sebron Harrison, $\mathrm{MD}^{2}$ \\ 1 Weill Cornell Physicians, New York, New York \\ ${ }^{2}$ New York Presbyterian Hospital, Weill Cornell Medical College, \\ New York, New York
}

\begin{abstract}
Address for correspondence Jeffrey Port, MD, Weill Cornell Physicians, 525 East 68th Street, M-404, New York, NY 10065

(e-mail: Jlp2002@med.cornell.edu).
\end{abstract}

Semin Intervent Radiol 2013;30:128-132

\begin{abstract}
Keywords

- lung cancer

- pulmonary nodule

- electromagnetic bronchoscopy (ENB)

Despite advances in technology and treatment options, lung cancer remains a deadly disease. National screening programs are being instituted in an attempt to discover lung cancer in high-risk individuals at an earlier stage. Such screening programs invariably discover small peripheral nodules that previously would not have been clinically apparent; the management of such lesions can be challenging. Current diagnostic options such as percutaneous biopsy are effective; however, they are hindered by their risk of morbidity such as pneumothorax. Electromagnetic bronchoscopy (ENB) is an emerging technology that allows the practitioner the ability to both sample and treat small peripheral pulmonary lesions. In experienced centers, ENB provides high rates of diagnostic yield for small lesions and a complication rate significantly lower than that of more conventional diagnostic modalities. Although there are current barriers to its widespread utilization (cost, specialized imaging, technical training), these obstacles will handled similarly to any other emerging technology and will likely not be long-term impediments to its use.
\end{abstract}

Objectives: Upon completion of this article, the reader will be able to identify the clinical utility and current limitations to electromagnetic navigational bronchoscopy.

Accreditation: This activity has been planned and implemented in accordance with the Essential Areas and policies of the Accreditation Council for Continuing Medical Education through the joint sponsorship of Tufts University School of Medicine (TUSM) and Thieme Medical Publishers, New York. TUSM is accredited by the ACCME to provide continuing medical education for physicians.

Credit: Tufts University School of Medicine designates this journal-based CME activity for a maximum of 1 AMA PRA Category 1 Credit $^{\mathrm{TM}}$. Physicians should claim only the credit commensurate with the extent of their participation in the activity.

Several recent trends highlight the importance of tissue diagnosis and localization of small pulmonary nodules. These trends include an increase in computed tomography (CT) lung cancer screening, minimally invasive thoracic surgery, sublobar lung resections, and the increased use of stereotactic body radiotherapy. A technology such as electromagnetic navigational bronchoscopy (ENB) is ideally suited to provide a platform for the treating physician to biopsy, localize, and even mark these small lesions for treatment.

Lung cancer remains a deadly disease, with 5-year survival rates $<20 \%$. The most significant reason for such poor survival rates is the late stage at presentation for most patients. Therefore, many strategies aimed at identifying and screening patients at high risk for lung cancer have been explored. Recently, the National Lung Cancer Screening Trial (NLST) reported a 20\% reduction in mortality in high-risk patients undergoing screening by computed tomography $(\mathrm{CT}){ }^{1,2}$ This will undoubtedly lead to an increase in patients presenting with small lung nodules; however, most of these nodules will be $<1 \mathrm{~cm}$ and benign. To avoid unnecessary procedures, it is important to have established protocols to deal with these small nodules. This approach will clearly require multidisciplinary review and diligent followup. Regardless of these perceived difficulties, at some point the ability to biopsy concerning lesions must be an integral part of any screening program.

Thoracic surgeons are increasingly relying on minimally invasive approaches for the resection of lung cancer. Numerous reports have highlighted the advantages of such modalities
Issue Theme Pulmonary Malignancies; Guest Editors, Bradley B. Pua, MD and David C. Madoff, MD, FSIR
Copyright (C) 2013 by Thieme Medical Publishers, Inc., 333 Seventh Avenue, New York, NY 10001, USA. Tel: +1(212) 584-4662.
Dol http://dx.doi.org/ 10.1055/s-0033-1342953. ISSN 0739-9529. 
over conventional approaches. They include a shortened length of stay, a decrease in pain and narcotic use, and a decrease in overall complication rate. Importantly, as our population ages, less invasive techniques offer these formerly "inoperable" patients the options of more limited resections (such as a wedge or segmental resection). However, the ability to perform sublobar resections by minimally invasive methods, either by a video-assisted approach or by the newly emerging robotic approach, relies on a preoperative tissue diagnosis. One persistent criticism of minimally invasive techniques is their limited ability to palpate and biopsy small or deep lesions intraoperatively. ENB has allowed for localization of these small deep lesions as well as the ability to obtain tissue diagnosis.

Conventional bronchoscopy, although relatively safe, has been associated with low diagnostic yields ( $\sim 25 \%)$, especially in small peripheral lesions. Yields for conventional bronchoscopy can be enhanced with the addition of fluoroscopy; fluoroscopic-enhanced bronchoscopy has diagnostic yields approaching 50\%., ${ }^{3,4}$ Combining low-dose multislice CT with bronchoscopy, for "virtual bronchoscopy," can further enhance yields. In most centers, patients with small peripheral lesions are biopsied by CT-guided percutaneous biopsy (CTFNA). CTFNA has improved diagnostic yields significantly and can approach 80 to $90 \%$ for appropriately selected lesions. The disadvantage to CTFNA is the requirement for expert interventional radiology services, $\mathrm{CT}$ time, and a percutaneous approach that has a 15 to $30 \%$ pneumothorax rate. The reported pneumothorax rate is even higher for smaller deeper lesions., ${ }^{5,6}$ A recent retrospective review of $>1000$ patients concluded that CT-guided lung biopsy carries a pneumothorax rate of $40 \%$, with $11 \%$ of all patients requiring chest tube placement. ${ }^{5}$

ENB has emerged as a useful diagnostic tool and a complement to the techniques just described. ENB combines conventional and virtual bronchoscopy, and it has allowed for sampling of lesions previously considered too small or peripheral for conventional bronchoscopic biopsy. It has extended the reach of conventional bronchoscopy out to the pleural surface. ENB can be performed in either a bronchoscopy suite or in the operating room under moderate sedation or general anesthesia.

The ENB system is composed of four components: a location guide (LG), a laptop computer with proprietary software for preprocedure planning, an electromagnetic mat placed under the patient, and an imaging tower (-Fig. 1). The procedure is divided into two phases: a

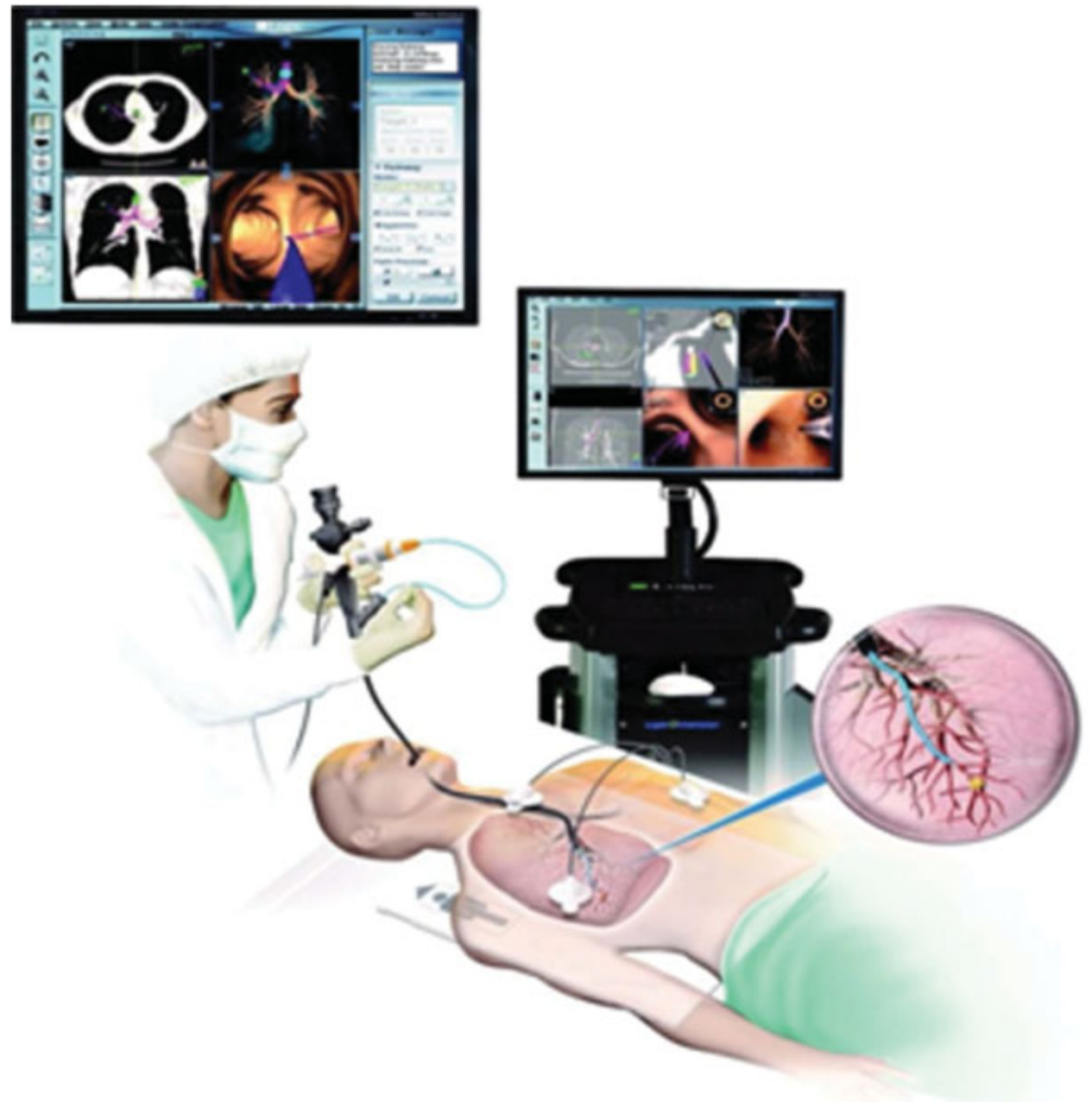

Figure 1 Four major components to electromagnetic navigational bronchoscopy: location guide, proprietary software used during planning phase, electromagnetic map, and imaging tower. 
planning phase performed prior to the procedure, and the navigational phase during which the lesion is sampled. During the planning phase, a thin-slice protocol CT of the chest, which shows the lesion of concern, is analyzed by proprietary software. The software then converts the patient's radiographic anatomy to virtual anatomy that will be navigated later during bronchoscopy. This process usually only takes minutes and can be easily performed just before the procedure. The patient is placed on the operating room table, which has a proprietary electromagnetic mat. This electromagnetic mat is used to link the LG probe (similar to GPS) of the ENB with the on-screen virtual bronchoscopy. Mapping takes place and involves a survey bronchoscopy, which the system links to the virtual bronchoscopy that was previously created. After mapping is complete, navigation can proceed.

The LG protrudes several centimeters out of the working channel of the bronchoscope. This LG is sensed by the proprietary software and displayed on screen in a virtual bronchoscopy format. Several different images are available to the bronchoscopist to facilitate navigation (-Fig. 2). The software generates colored "guides" or "roadmaps" in the virtual bronchoscopy to aid in navigation; however, the bronchoscopist does not have to follow such guides if he or she deems another route more appropriate. As the navigation proceeds closer to the lesion, the probe may be turned or flexed as needed to traverse sharp angles in the smaller airways. As the lesion is approached, the software displays the distance from the tip of the probe to the lesion. Once the lesion is reached, the bronchoscope is wedged into the airway, and the working channel is locked into position. A variety of options for sampling the lesion are available and may be passed into the working channel for sampling. These include needle aspiration, brush biopsy, and forceps. The ability to obtain multiple passes and even forceps biopsy increases the likelihood of obtaining adequate samples for biomarker evaluation.

Several recent studies have sought to identify factors that may improve the success of ENB biopsy. An excellent recent review of such studies by Leong and colleagues ${ }^{7}$ identified certain factors from the current literature thought to improve the success of biopsy. One such factor was the finding of the so-called bronchus sign on preprocedure CT. A bronchus sign occurs when a bronchus leads directly to a peripheral lesion. One such study ${ }^{8}$ that specifically looked at the impact of the bronchus sign on ENB concluded that the presence of a bronchus sign was associated with significantly higher diagnostic yield; overall diagnostic yield was $67 \%$. Of these positive results, $>80 \%$ had a bronchus sign.

Rapid on-site cytopathologic examination (ROSE) has also been suggested to improve diagnostic yield. ${ }^{9,10}$ Although no current standard practice exists, we believe that ROSE greatly facilitates diagnostic yield, and our practice is to have on-site immediate cytopathologic review of the specimen sampled. Immediately after sampling, a cytopathologist reviews the specimen; navigation and biopsy are repeated until there is confirmation the lesion has been sampled. Size of the lesion clearly plays a role in diagnostic yield. In our experience, lesions $>2 \mathrm{~cm}$ significantly increase yield. Similarly, location of a lesion may have an impact. Upper lobe and posteriorly

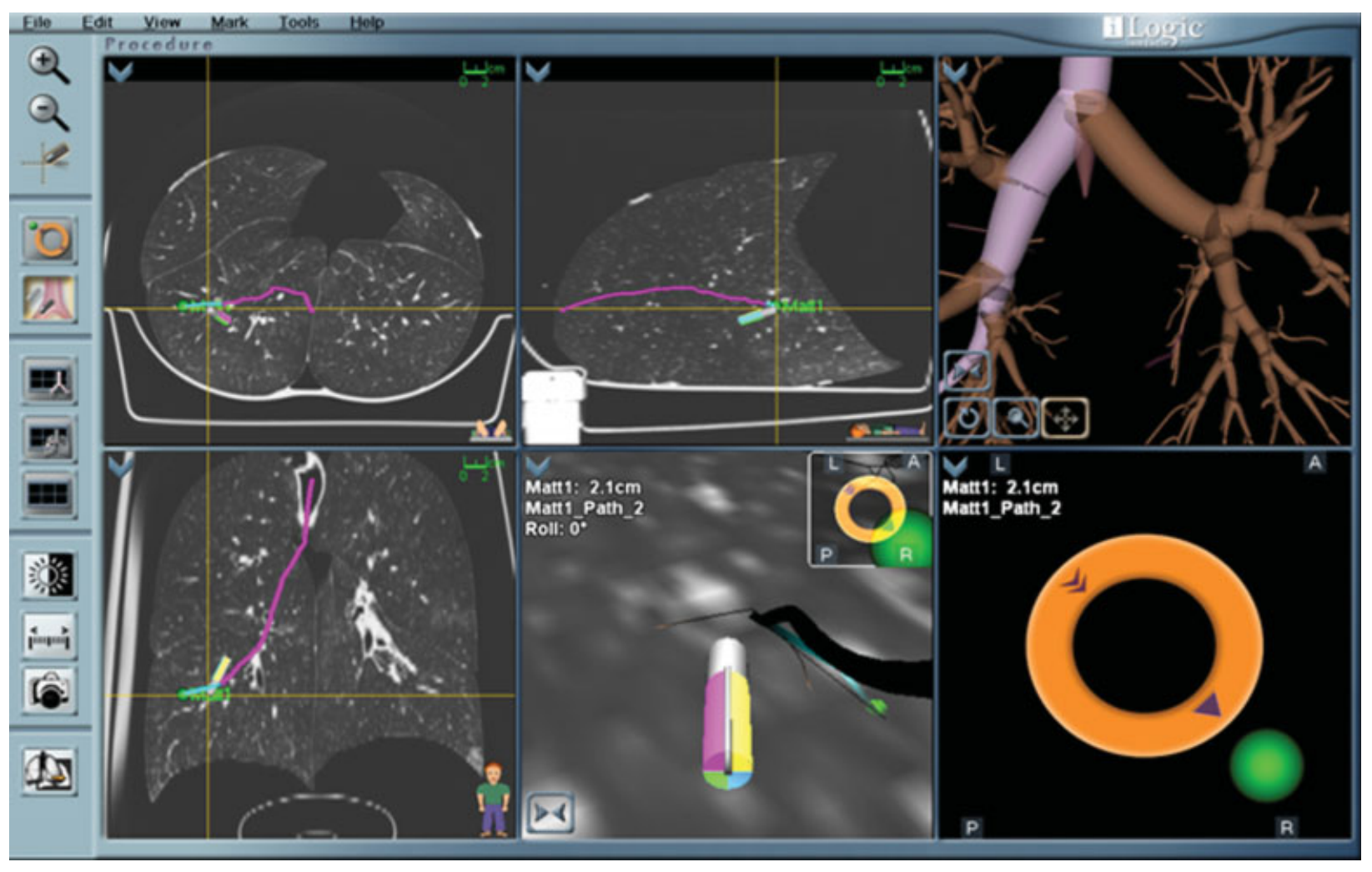

Figure 2 Multiple images aid in diagnostic yield during the navigational phase of electromagnetic navigational bronchoscopy. 
located lesions have traditionally presented challenges to ENB. This has recently been addressed with the advent of a new flexible catheter tip that greatly facilitates traversing the sharp angles required in these locations. Although supported by some case reports and series as an aid to improving diagnostic yield, the use of general anesthesia and fluoroscopy is not mandatory and can be utilized according to the experience and comfort level of the bronchoscopy team.

Certain impediments to widespread use of ENB have been noted and are similar to those associated with any new technology. The learning curve for ENB has been considered significant. Although the feel of ENB is considerably different from that of conventional bronchoscopy, the technology lends itself to ease of training both in real-time applications and in simulation. The learning curve is primarily related to learning the new technology; however, once this is mastered even small lesions may be amenable to sampling. An early study concluded the diagnostic yield of ENB to be $63 \%$ for small (mean: $2.3 \mathrm{~cm}$ ), peripheral $(1.4 \mathrm{~cm}$ from parietal pleura) lesions. As our skill with ENB has increased and with the recent improvements in technology, diagnostic yield in our practice has approached $80 \%$.

Costs, although significant, are not prohibitive, and most American insurance companies currently cover ENB. One area that should be addressed in any discussion of costs is that of the preprocedure CT. Current software requires a thin-slice chest CT on "hard copy." Because patients often receive a CT scan prior to any consideration of ENB, these criteria are often lacking, and a repeat CT is required. Obviously, the cost of repeated $\mathrm{CT}$ and concerns of increasing radiation exposure sometimes prohibit proceeding with ENB. However, with improvements in proprietary software and increased awareness of ENB by practitioners, we hope these issues will become less of a deterrent.

Major complications are rare and limited primarily to bleeding, hemoptysis, and pneumothorax. The pneumothorax rate has recently been reported as high as $7 \%,{ }^{7}$ although our experience shows a pneumothorax rate $<1 \%$. Hemoptysis and hemothorax, although reported in the literature, are exceedingly rare. Most patients may be discharged home after appropriate chest X-ray and postanesthesia monitoring are completed. The relatively low rate of complications from ENB is certainly one of its most compelling features, both from a patient care and health care cost standpoint.

Although the initial interest in ENB was centered on its ability to sample small peripheral lung lesions, other diagnostic and therapeutic measures are available. One particularly frustrating clinical case is a small peripheral groundglass opacity that has eluded diagnosis by percutaneous biopsy. When conventional measures were nondiagnostic, surgeons often had to attempt wedge biopsy on these lesions that may not be palpable at the time of surgery. This often leads to multiple wedge biopsies under general anesthesia or a much larger than desired lung resection to include the concerning lesion. The use of ENB in such cases allows the surgeon to mark the lesion prior to surgery, therefore limiting the size of resection. After the lesion is located during navigation, methylene blue is injected near the lesion. The surgeon resects the marked area during the same anesthesia, and intraoperative frozen sectioning confirms successful resection. This greatly aids lung preservation in patients at risk for multiple future resections. One other and particularly exciting area of interest is the use of ENB to mark lesions for stereotactic radiation treatment. With ongoing prospective trials evaluating the efficacy of stereotactic radiation therapy in the treatment of early stage localized lung cancer, ${ }^{11}$ the utilization of ENB to mark lesions for such therapy will continue to increase.

ENB is ideally situated as a therapeutic platform as well. Microwave, radiofrequency ablation, cryotherapy, and even radiation may soon be offered through this modality. Physicians will be able to obtain a tissue diagnosis and potentially treat during the same procedure and anesthesia.

As more patients undergo screening for lung cancer, management strategies for small peripheral lung lesions will advance. Prior options, although useful in many situations, have been thwarted by low diagnostic yield or high complication rates. ENB is an emerging technology that has attempted to address both of these problems, as well as to provide new therapeutic options previously unavailable. It will undoubtedly continue to evolve into a major therapeutic and diagnostic modality useful in the management of lung disease.

\section{References}

1 Aberle DR, Adams AM, Berg CD, et al; National Lung Screening Trial Research Team. Reduced lung-cancer mortality with low-dose computed tomographic screening. N Engl J Med 2011;365(5): 395-409

2 Henschke CI, McCauley DI, Yankelevitz DF, et al. Early Lung Cancer Action Project: overall design and findings from baseline screening. Lancet 1999;354(9173):99-105

3 Hautmann $\mathrm{H}$, Henke MO, Bitterling H. High diagnostic yield from transbronchial biopsy of solitary pulmonary nodules using lowdose CT-guidance. Respirology 2010;15(4):677-682

4 Tsushima K, Sone S, Hanaoka T, Takayama F, Honda T, Kubo K. Comparison of bronchoscopic diagnosis for peripheral pulmonary nodule under fluoroscopic guidance with CT guidance. Respir Med 2006;100(4):737-745

5 Hiraki T, Mimura H, Gobara H, et al. Incidence of and risk factors for pneumothorax and chest tube placement after CT fluoroscopyguided percutaneous lung biopsy: retrospective analysis of the procedures conducted over a 9-year period. AJR Am J Roentgenol 2010;194(3):809-814

6 Li H, Boiselle PM, Shepard JO, Trotman-Dickenson B, McLoud TC. Diagnostic accuracy and safety of CT-guided percutaneous needle aspiration biopsy of the lung: comparison of small and large pulmonary nodules. AJR Am J Roentgenol 1996;167(1):105-109

7 Leong S, Ju H, Marshall $\mathrm{H}$, et al. Electromagnetic navigation bronchoscopy: a descriptive analysis. J Thorac Dis 2012;4(2): 173-185

8 Seijo LM, de Torres JP, Lozano MD, et al. Diagnostic yield of electromagnetic navigation bronchoscopy is highly dependent on the presence of a Bronchus sign on CT imaging: results from a prospective study. Chest 2010;138(6):1316-1321

9 Lamprecht B, Porsch P, Pirich C, Studnicka M. Electromagnetic navigation bronchoscopy in combination with PET-CT and rapid on-site cytopathologic examination for diagnosis of peripheral lung lesions. Lung 2009;187(1):55-59 
132 Electromagnetic Navigational Bronchoscopy Port, Harrison

10 Wilson DS, Bartlett RJ. Improved diagnostic yield of bronchoscopy in a community practice: combination of electromagnetic navigation system and rapid on-site evaluation. J Bronchol 2007;14:227-232

11 National Cancer Institute, American College of Surgeons Oncology Group, Radiation Therapy Oncology Group. A randomized phase III study of sublobar resection (+/- brachytherapy) versus stereotactic body radiation therapy in high risk patients with stage i nonsmall cell lung cancer (NSCLS). In: ACOSOG Protocol Z4099, RTOG Protocol 1021. St. Louis, MO: Washington University School of Medicine; 2011 